

\section{Implantologie war Stargast in Cannes}

Über 1.500 Teilnehmer aus aller Welt erlebten auf der MIS Global Conference in Cannes vom 6. bis 9. Juni unter dem Motto „360 Implantology“ ein internationales Fortbildungshighlight. Das prall gefüllte Vortragsprogramm thematisierte alle Herausforderungen der Implantologie.

In Vorträgen und Hands-on-Workshops gaben die etwa 35 Referenten ihr Fachwissen weiter. Als Symbiose zwischen Kunst und Wissenschaft bezeichnete Dr. Henriette Lerner aus Baden-Baden im Rahmen ihres Vortrags die zahnärztliche Implantologie. Dr. Maurice A. Salama, Lehrbeauftragter für Parodontologie in Pennsylvania und Georgia, befasste sich mit Trends, Technologien und Techniken der implantologischen Zahnmedizin. Neu- este Erkenntnisse zum Knochenaufbau präsentierten unter anderem Prof. Michal Peleg von der Medizinuniversität Miami und Dr. Matthias Plöger aus Detmold. Mit Dr. Paolo Cardelli, Dr. Joile Alvarez Cantoni, Dr. James Collins und Dr. Emilio Mateo stellten die Gewinner der MIS Clinical Cases Competition ihre beeindruckenden Fallstudien zu dem Thema „The combined use of MIS dental implants and BONDBONE for immediate procedures in the esthetic zone“ vor. Dr. Nachum Samet, einst Lehrbeauftragter für restaurative Zahnheilkunde an der Harvard School of Dental Medicine und nun in der Abteilung für Forschung und Entwicklung bei MIS tätig, sprach darüber, wie bei MIS unter dem Motto „Make it simple“ Innovationen

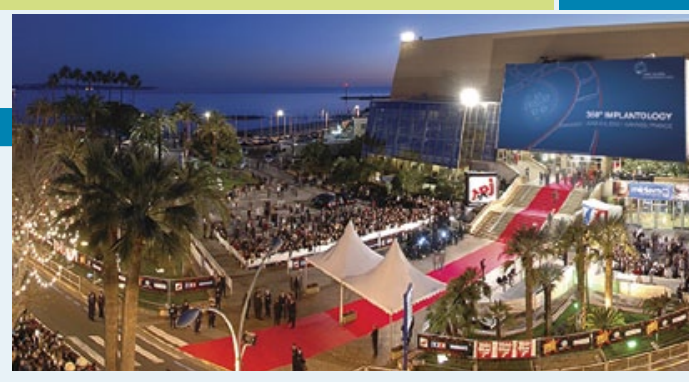

für die Implantologie entwickelt werden. Im Rahmen der Produktpräsentationen von MIS konnten die Kongressteilnehmer zudem mehr über das Implantat $\mathrm{C}$, das die Lücke zwischen Primär- und Sekundärstabilität minimiert, und über die 4 Hauptimplantate M4, UNO, SEVEN und LINK erfahren.

Es darf sich 2015 auf die nächste traumhafte Location gefreut werden, wenn es wieder heißt „360 Implantology“.

Nach einer Pressemitteilung der

MIS Implants Technologies, Minden

Internet: www.mis-customers.com/de

\section{Implantologie}

\section{Neue Implantatgeneration im eigenen Themenpark}

BIOMET $3 \mathrm{i}$ hat im Rahmen des „11 ${ }^{\text {th }}$ International Symposium on Periodontics and Restorative Dentistry“-Meetings in Boston das neue $3 \mathrm{i}$ T3 ${ }^{\circledR}$ Implantat vorgestellt. Kongressbesucher aus der ganzen Welt erfuhren auf ihrer Tour durch den eigens eingerichteten Themenpark „3i T3 Preservation Destination“, wie das neue Implantat designt wurde, um mit dem Erhalt von Hart- und Weichgewebe nachhaltige ästhetische Ergebnisse zu ermöglichen. Während des Symposiums gab es außerdem die Gelegenheit, an einem „VIP
German Dinner“ teilzunehmen, zu dem Bart Doedens, Präsident von BIOMET 3i, und Dr. Ron Nevins eingeladen hatten. Das Dinner gab den Rahmen für intensive Gespräche in einem kleinen exklusiven Kreis von deutschsprachigen Zahnärzten sowie mit den renommierten Parodontologen Professor Dr. Harold S. Baumgarten und Dr. Richard Lazzara, Unternehmensgründer von BIOMET 3i.

Nach einer Pressemitteilung der BIOMET 3i Deutschland GmbH, Karlsruhe

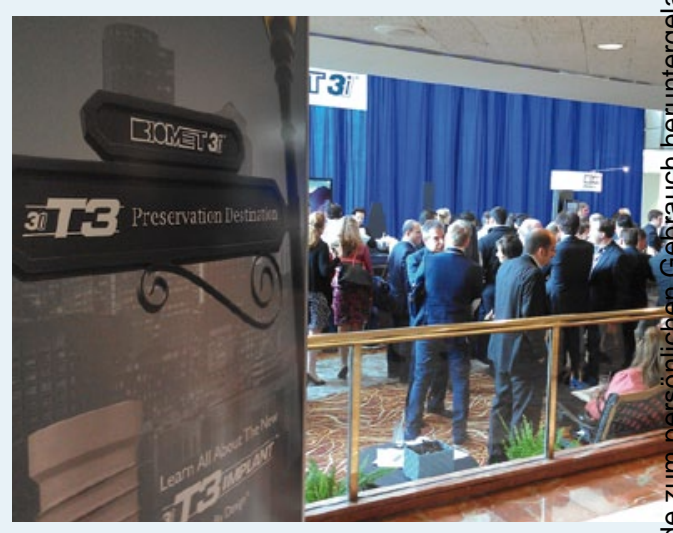

Für die Zahnmedizin steht nun ein nach dem Vorbild der Natur geformter und dreidimensional aus Keramik in Dentin und Schneide industriell geschichteter Kronenrohling zur Verfügung. Die Kronen werden volldigital inhouse oder fremdgefertigt. Das Portfolio für Front- und Seitenzähne umfasst ein breites Spektrum an Formen, Farben und Größen - passend für nahezu jede Indikation. Einfach, schnell und effizient ist die Anwendung der priti ${ }^{\circledR}$ crowns im Laboralltag. Dass das Gleiche für das Erlernen der Verfahrens- weise gilt, zeigen nun 3 Anwendervideos. Die Filme stehen in den Sprachen deutsch und englisch zum Download bereit unter http://www.pritidenta.com/cms/ nid/135/news.html.

priti ${ }^{\circledR}$ crown-CAD-Design: Die Arbeitsschritte für das CAD-Design mit 3Shape und exocad können anhand der Videos step by step nachvollzogen und als Schulungsvideos für die eigene Arbeit oder für Mitarbeiter genutzt werden. Die Livemitschnitte der Videos beweisen, dass das CAD-Design einer Einzelkrone mit der priti ${ }^{\circledR}$ crown nur ca. 5 Minuten benötigt.

priti ${ }^{\circledR}$ crown-Finalisierung: Mit der priti ${ }^{\circledR}$ crown können von der Einzelkrone bis zu Full-Mouth-Restaurationen viele Indikationen abgedeckt werden. ZTM Axel Seeger zeigt im Video Möglichkeiten und die wichtigsten Handgriffe, um priti ${ }^{\circledR}$ crowns für ästhetischen Zahnersatz zu nutzen.

Nach einer Pressemitteilung der pritidenta $\mathrm{GmbH}$, Leinfelden-Echterdingen Internet: www.pritidenta.com 
Fortbildung

\section{Gelungener Auftakt der Ladies Nights} in Deutschland

Der Implantathersteller Implant Direct lud Zahnärztinnen und Implantologinnen am 3. Juli zur Ladies Night nach Düsseldorf ein. Während des zweistündigen Events referierte die Oralchirurgin Dr. Friederike Ratschow, MSc, zum Thema „Sinuslift - Wann und wie?“. Der Schwerpunkt ihres Vortrags lag auf der strukturierten Behandlung im schwierigen OberkieferSeitenzahnbereich. Die Implantologin beschrieb anhand vieler anschaulicher und interessanter Fälle das Vorgehen beim lateralen sowie beim internen Sinuslift. Eine klare Empfehlung von Ratschow war, im Rahmen der präoperativen Planung und Diagnostik unbedingt eine DVT-Aufnahme anfertigen zu lassen. Einige anatomische Besonderheiten wären nur so zu diagnostizieren. Abschließend hatte Ratschow noch eine gute Nachricht für die Teilnehmerinnen: „Die Erfolgsaussichten

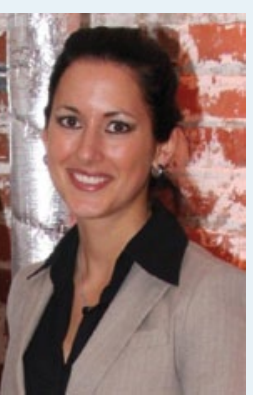

beim Sinuslift (lateral sowie intern) liegen bei über 95\%.“ Im Anschluss an den Fachvortrag nutzten die Zahnärztinnen und Implantologinnen noch die Möglichkeit, die Sinuslift-Expertin zu Fällen und Herausforderungen $\mathrm{zu}$ befragen. „Das Feedback der Teilnehmerinnen zeigt, dass unser Konzept angenommen wird“, so Manuela Lutiger, Head of Marketing bei Implant Direct.

Die nächste Ladies Night findet am 11. September bei Frau Tonis Parfum in Berlin statt. Interessierte Zahnärztinnen und Implantologinnen erhalten weitere Informationen unter der Infoline 008004030 4030 (kostenfrei), Fax +41 445678101 oder per E-Mail an events@implantdirect.eu. Eine Anmeldung ist erforderlich, die Teilnehmerzahl ist begrenzt.

Nach einer Pressemitteilung der Implant Direct Sybron Europe AG, CH-Zürich

\section{Studie}

\section{Dental Advisor vergab Bestnote}

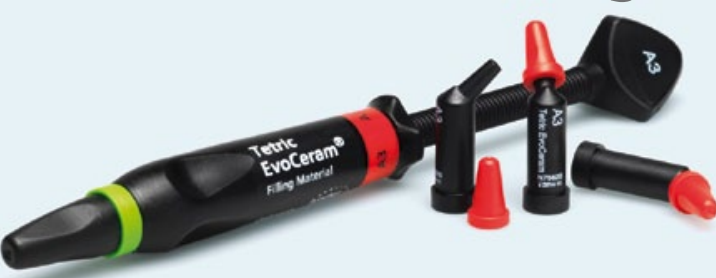

Das unabhängige US-amerikanische Testinstitut „The Dental Advisor“ hat das Komposit Tetric EvoCeram von Ivoclar Vivadent über einen Zeitraum von 5 Jahren getestet und mit der Bestnote 5 ausgezeichnet. Zudem hat das Testinstitut dem Komposit das Prädikat „Top Long Term Performer Composite 2013" verliehen.

Für die Studie wurden insgesamt 800 Füllungen gelegt. Zum Recall kamen 615 Patienten. Davon hatten 96\% Seitenzahn- und 4\% Frontzahnfüllungen. 63\% der Füllungen wurde mit dem Adhäsiv AdheSE von Ivoclar Vivadent gelegt. Bei den restlichen Füllungen kamen unterschiedliche Adhäsive zum Einsatz. In der Studie erreichte das lichthärtende, universelle Nanohybrid-Komposit eine kli-

nische Bewertung von 97\% nach 5 Jahren. Aus der Studie ging hervor, dass Tetric EvoCeram sehr resistent gegenüber Bruch bzw. Chipping ist. Zudem war die Ästhetik der Restaurationen beim Recall ausgezeichnet: Von 615 untersuchten Restaurationen wurden 572 (93\%) mit der Bestnote 5 (ausgezeichnet) bewertet. 38 Restaurationen $(6,2 \%)$ erhielten die Note 4 (sehr gut) und nur 5 (0,8\%) wurden als schlecht beurteilt. Das Testinstitut zieht daraus folgendes Fazit: „Tetric EvoCeram ist ein Komposit mit ausgezeichneter Handhabung, das für ausgezeichnete Ergebnisse sorgt. Es ist stopfbar, klebt nicht, lässt sich gut modellieren sowie einfach ausarbeiten und polieren.“

Quellen: The Dental Advisor, Vol 29, Nr. 8, Oktober 2012/Dental Advisor Vol. 30, Nr. 01, Januar-Februar 2013

Nach einer Pressemitteilung der Ivoclar Vivadent AG, FL-Schaan Internet: www.ivoclarvivadent.com

\section{Online-Angebot}

\section{ab sofort passend für jedes Gerät}

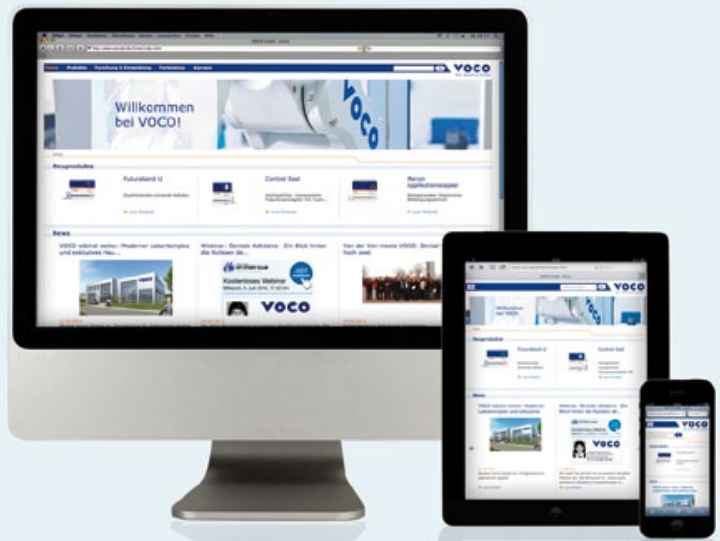

VOCO bietet den Nutzern seiner Website einen besonderen Komfort an: Ab sofort ist die Firmenhomepage voco.de im Responsive-Webdesign online und stellt sich somit immer automatisch auf das Gerät ein, mit dem sie betrachtet wird. Mit der Umstellung reagiert VOCO auf die zunehmende Nutzung von mobilen Geräten. Ob auf dem Smartphone, dem Tablet oder doch am großen Desktop-Bildschirm: Dank des Reponsive-Design lässt sich das Browserfenster stufenlos skalieren, während die Inhalte sich der entsprechenden Breite anpassen. Der grafische Aufbau von voco.de orientiert sich an den individuellen Anforderungen des jeweiligen Gerätes und stellt alle Inhalte optimal aufbereitet, leicht leserlich und übersichtlich dar. Noch nie war es so einfach, die volle Bandbreite an Informationen abzurufen - ganz gleich, wie groß das Display ist. Zugleich präsentiert sich auch das Layout der Seite frischer und übersichtlicher: Die Inhalte sind noch klarer strukturiert und einfach zu überschauen. Auf der neuen Startseite warten nur die neusten VOCO-Produkte und die wichtigsten News - so fällt die Orientierung leichter. Weiterführende Informationen lassen sich rasch abrufen, während das Gesamtmenü dabei stets den Nutzungspfad angibt und so für Orientierung sorgt. Größere Bilder, eine größere Schrift und ein aufgelockertes Layout garantieren neben dem Responsive-Design noch mehr Benutzerfreundlichkeit.

Nach einer Pressemitteilung der voco GmbH, Cuxhaven

Internet: www.voco.de 


\section{Das Pipe Augmentation-System}

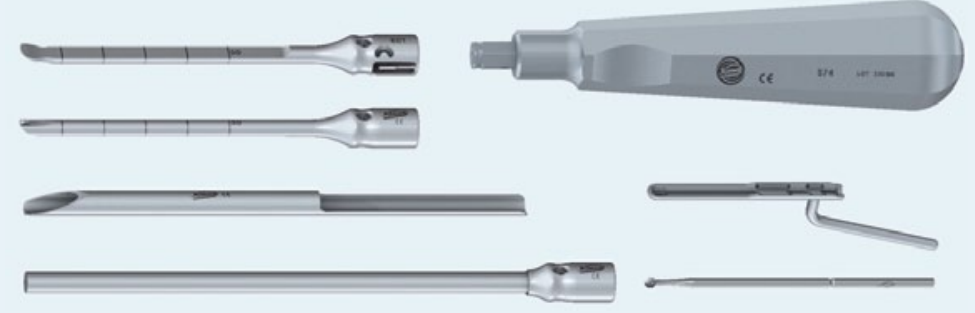

Dr. Dr. Karl-Heinz Heuckmann und Prof. Dr. Klaus-Ulrich Benner entwickelten eine minimal-invasive Methode zur Erhöhung und Verbreiterung atropher Kieferkämme durch subperiostale Augmentatauflagerung: die sog. Stollentechnik. Dabei wird Augmentationsmaterial subperiostal eingebracht, das aushärtet, sobald es in Kontakt mit Körperflüssigkeit tritt. Die minimalinvasive Methode erlaubt eine Modellierung des Augmentats nach Einbringung in den Stollen, ist zeitsparend, schnell vom Operateur erlernbar und liefert gute präimplantologische Voraussetzungen für eine sichere Implantatbettgestaltung. Das Verfahren wird von Komet durch die ideal darauf abgestimmten Instrumente im Pipe Augmentation-System unterstützt: blendfreie Oberflächen, kompatibler Wechselhandgriff, ergonomische Formen, Inserttray bzw. Sterilcontainer und beste Führung und Kontrollierbarkeit spiegeln die bewährte Komet-Qualität wider.

Nach einer Pressemitteilung der

Komet Dental Gebr. Brasseler GmbH \& Co KG, Lemgo

Internet: www.kometdental.de
Verpackungänderung

Bewährte Qualität in neuem Design

Im Rahmen des neuen Markenauftritts, unter dem Kreussler Pharma seine DYNEXAN ${ }^{\circledR}$-Produkte seit Beginn des Jahres als Therapiekonzept bei Schmerzen und Entzündungen im Mund vermarket, bekommt DYNEXAN PROAKTIV ${ }^{\circledR} 0,2 \%$ CHX ein neues Gesicht.

Die alkoholfreie Mundspüllösung

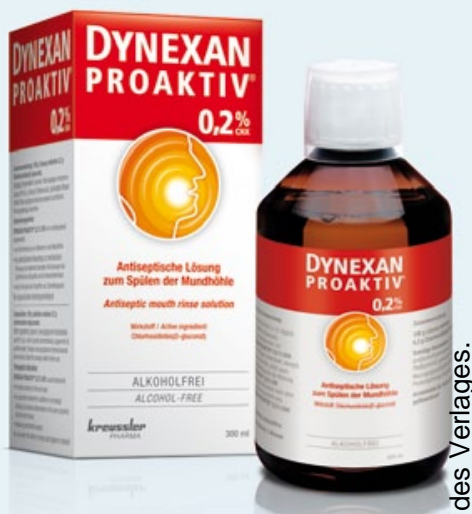
mit 0,2\% Chlorhexidin zur Keimreduktion - sowohl in der Zahnarztpraxis als auch in der Nachsorge zuhause - wird auch weiterhin in ihrer bewährten Rezeptur angeboten, es ändert sich nur das Design. Erstmalig ist die Verpackung zweisprachig, in Deutsch und Englisch gekennzeichnet. Kreussler Pharma bietet somit ein Produkt, das von Zahnärzten, Ärzten und Apothekern auch an fremdsprachige Patienten empfohlen werden kann und die Anwendung in diesem Bereich maßgeblich erleichtert.

Bitte beachten Sie: Die Umstellung auf das neue Verpackungsdesign erfolgt in den nächsten Wochen. Produkte in der ursprünglichen Verpackung bleiben weiterhin im Handel, eine Rücknahme bzw. ein Umtausch sind nicht vorgesehen.

Nach einer Pressemitteilung der

Chemische Fabrik Kreussler \& Co. GmbH, Wiesbaden

Internet: www.kreussler.com

\section{Clever investieren}

DVTs bieten viele Vorteile für Diagnostik und Therapie - doch aufgrund der hohen Investition in die 3D-Röntgensysteme schrecken Behandler oft vor einer Anschaffung zurück und entscheiden sich stattdessen zuerst für ein digitales Panoramasystem. Dabei ist der Preissprung zwischen einem reinen Panoramasystem und dem CS 9000 3D, das zusätzlich zum Panoramaröntgen die volle DVT-Funktionalität bietet, aktuell so gering wie nie. Der Imaging-Spezialist Carestream Dental bietet in seiner Sommeraktion das CS 9000 3D ab dem 1. Juli bei allen teilnehmenden Händlern zum Sonderpreis von nur 39999,-€ an. Der digitale Allrounder kostet damit nur wenig mehr als ein hochwertiges Panoramaröntgensystem ohne DVT - und die Praxis ist mit dem CS 9000 3D gut für die Zukunft gerüstet. Wer die Anschaffung eines digitalen Panoramaröntgensystems plant, sollte diese einmalige Gelegenheit nutzen und gleich zur kombinierten Panorama- und 3D-Lösung greifen. Das System ist eine Kombination aus 2D-Panorama- und 3D-DVT-Technologie. Angezeigt werden können axiale, koronare, sagittale und auch benutzerdefinierte Schichten. Die dreidimensionale Rekonstruktion bietet darüber hinaus eine absichernde realistische Ansicht der Zahnstrukturen für sichere Diagnosen. Wird eine Modalität auf dem Computer ausgewählt, wechselt die Einheit automatisch zwischen 3D- und Panoramamodus - ein manuelles Switchen des Sensors wird damit überflüssig. Das System verfügt über einen Wirkungsbereich $(50 \times 37 \mathrm{~mm}$ FOV) für hochauflösende Aufnahmen mit einer Kantenlänge (oder Mindeststärke) von $0,076 \mathrm{~mm}$. Aufgrund seiner hohen Auflösung eignet sich das CS 9000 3D hervorragend für beinahe alle lokalen Zahnbehandlungen, auch für sehr anspruchsvolle Eingriffe, wie Wurzelkanalbehandlungen und Einzelimplantate. Durch den effizienten Wirkungsbereich reduziert sich auch die Strahlungsbelas-

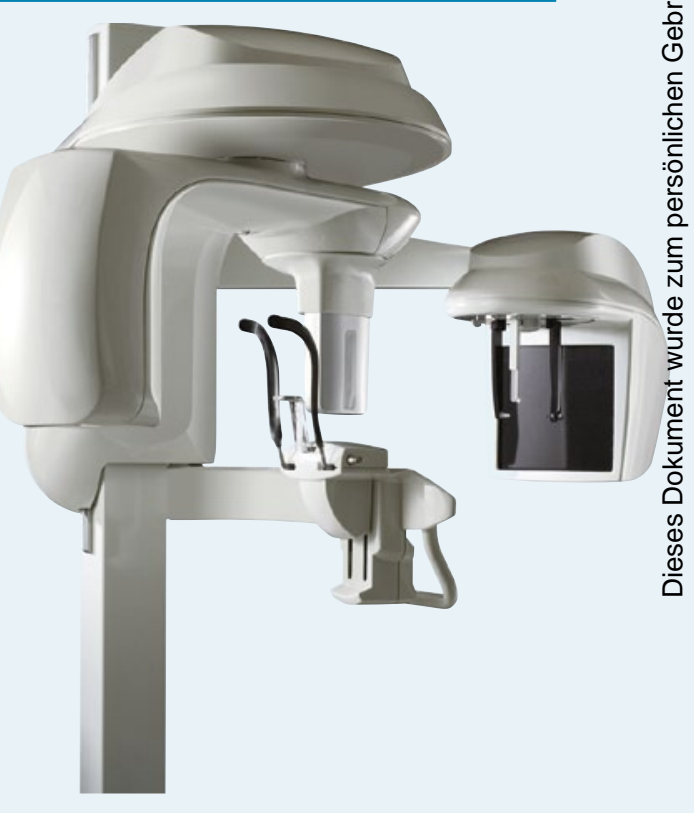

tung im Vergleich zu 3D-Systemen mit einem größeren Bildbereich.

Nach einer Pressemitteilung der

Carestream Health Deutschland $\mathbf{G m b H}$,

Stuttgart

Internet: www.carestreamdental.de 


\section{Erfolgreiche Lösungen richten sich am Behandlungsablauf aus}

Von der patientenorientierten Aus- und Fortbildung bis hin zur ergonomischen Gestaltung von Arbeitsplätzen, Geräten und Instrumenten: Das Unternehmen Morita setzt bewusst auf anwendergerechte Innovationen und Verbesserungen, die ganz auf die Bedürfnisse der jeweiligen Zielgruppe zugeschnitten sind. Ob neuartige Ausbildungssysteme, wie der Patientenroboter SIMROID, oder umfassende Systemlösungen für Endodontie, Implantologie und Kieferorthopädie. Intuitive Bedienungsschritte für das Behandlerteam in der Diagnostik, Implantologie, Endodontie und Kieferorthopädie zeichnen die Komplettlösungen aus.

Das Verständnis der Abläufe für die Endodontie setzt beispielsweise bei der exakten Darstellungsmöglichkeit durch die
Röntgengeräte für Einzelzahnaufnahmen und Digitale Volumentomografen (DVT) zur Darstellung komplexer Strukturen an, wobei alle Systeme gemäß dem ALARAPrinzip eine sehr geringe Strahlenbelastung aufweisen. Den fließenden Übergang von der Diagnose zur Therapie unterstützt die ergonomische Behandlungseinheit Soaric. Sie ist für endodontische Behandlungen entwickelt worden, setzt auf intuitive Greifwege für die Instrumente, integriert intelligente Ablagemöglichkeiten und bietet mit einem innovativen Design ein hohes Maß an Liegekomfort für den Patienten. Auch für die weiteren Arbeitsschritte - Trepanation, Aufbereitung, Behandlung und Kontrolle - bietet Morita Instrumente und Systemlösungen für einen runden und erfolgreichen Ablauf.

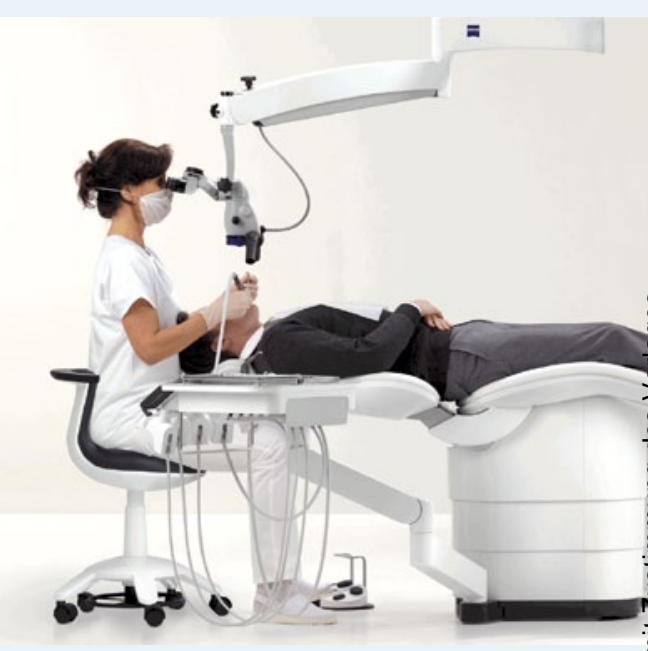

Nach einer Pressemitteilung der

J. Morita Europe GmbH, Dietzenbach

Internet: www.morita.com/europe
Regenerative Verfahren

\section{Gewinnung von}

\section{autologem Knochen}

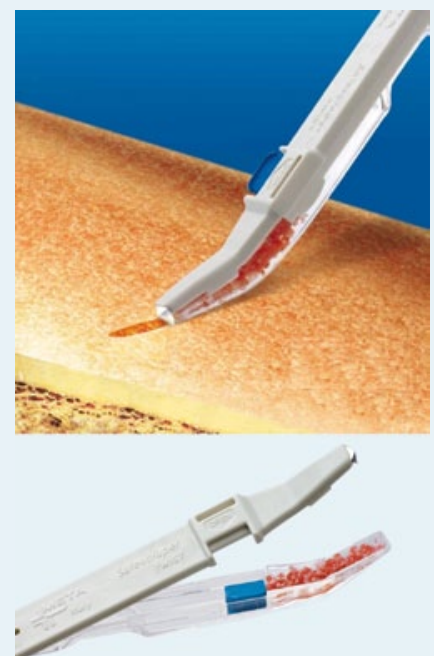

Autologer Knochen gilt bei regenerativen Verfahren in der Zahnmedizin auch heute noch als Goldstandard. Die intraorale Gewinnung von kortikalen Knochenspänen gelingt mittels dem originalen Safescraper ${ }^{\circledR}$-Twist medizinisch sicher, einfach und schnell. Die speziell geformte, extrem scharfe Klinge erlaubt je nach Druckausübung die Sammlung kleiner und großer Mengen kortikaler Knochenspäne - unter Wahrung maximaler Zellvitalität (speziell von Osteozyten, Osteoblasten und Osteoklasten mit durchschnittlicher Vitalität von $45-72 \%$ ).

Der gesammelte Knochen ist bereits mit Blut vermischt und lässt sich aus der aseptischen, abnehmbaren Kammer direkt in den vorgesehenen Defekt übernehmen. Dank des mitaufgenommenen Blutes gewinnt das Augmentat bereits kurze Zeit nach der Entnahme eine hohe biologische Plastizität. Die gesamte Masse lässt sich mittels einer Pinzette als Objekt gut bewegen und platzieren. Die sterilen Instrumente arbeiten minimalinvasiv, sind gebrauchsfertig verpackt und auch für den mehrmaligen Gebrauch in einer Sitzung pro Patient zu verwenden. Eine Safescraper-Twist-Kammer sammelt ca. $2,5 \mathrm{~cm}^{3}$ (gebogene Variante) bzw. $3 \mathrm{~cm}^{3}$ (gerade Variante) Knochenmaterial.

Nach einer Pressemitteilung der

Zantomed GmbH, Duisburg

Internet: www.zantomed.de

\section{Fortbildung \\ Komposit-Schichttechnik inmitten italienischer Sportwagen}

Ferraris sind der Inbegriff von Eleganz, Stil und makellosem Design. Doch dass die Italiener wahre Ästheten sind, zeigt sich nicht nur in ihren Sportwagen: Sie sind auch Meister der direkten Füllungstherapie in der Zahnheilkunde und beherrschen es, durch die geschickte Kombination ver-

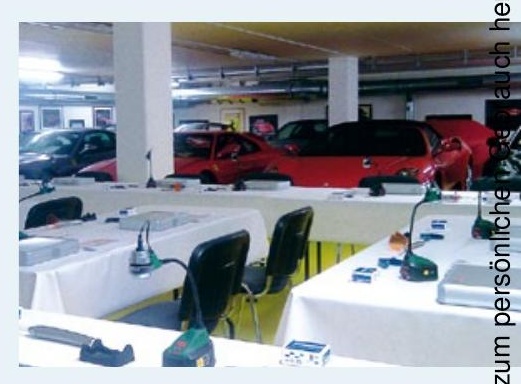
schiedener Massen Zähne naturgetreu nachzubilden. Nun gibt es ein Konzept zur schnellen Herstellung ästhetischer direkter Restaurationen selbst im hektischen Praxisalltag: Style Italiano. Seit einigen Monaten können Zahnärzte das von den Italienern Prof. Dr. Angelo Putignano und Dr. Walter Devoto entwickelte Konzept am Beispiel des Füllungsmaterials Filtek Supreme XTE Universal Komposit von 3M ESPE in Workshops erlernen. Neu ist die von Kai Wagner (Verkaufsrepräsentant bei 3M ESPE) stammende Idee, die Seminare in echt italienischem Ambiente zu veranstalten: Am 4. Juli 2013 lud 3M ESPE in die Verkaufsräume eines Ferrari-Händlers nach Kassel ein, um zwischen den Sportwagen die Kunst der einfachen Komposit-Schichttechnik zu vermitteln. Prof. Dr. Claus-Peter Ernst (Universitätsmedizin Mainz) erläuterte den Teilnehmern detailliert, worauf es in der ästhetischen Füllungstherapie und bei dem Zweifarb-Schichtkonzept Style Italiano ankommt. Die Reaktion der Teilnehmer auf die praxisnahe Wissensvermittlung im stilvollen Ambiente war überwältigend. Weitere Informationen unter www.3MESPE.de/Veranstaltungen.

Nach einer Pressemitteilung der 3M Deutschland GmbH, Seefeld 